\title{
NY-ESO-1/GLA-SE Vaccine ID-G305
}

National Cancer Institute

\section{Source}

National Cancer Institute. NY-ESO-1/GLA-SE Vaccine ID-G305. NCI Thesaurus. Code C113432.

A cancer vaccine composed of a recombinant form of the tumor antigen NY-ESO-1 and glucopyranosyl lipid adjuvant (GLA)-stable emulsion (GLA-SE), with potential antineoplastic and immunomodulating activities. Upon intramuscular injection, the adjuvant portion of the NY-ESO-1/GLA-SE vaccine ID-G30 binds to toll-like receptor subtype 4 (TLR-4) expressed on dendritic cells (DCs), monocytes, macrophages and B cells. The activated DCs present the NY-ESO-1 antigen to Th1 CD4 T-lymphocytes. This leads to the induction of cytotoxic T lymphocytes (CTLS) and the killing of NY-ESO-1-expressing tumor cells. This vaccine also induces specific antibody responses and increases the production of inflammatory cytokines. 